\title{
Effects of Head Size on the Mechanical Properties of Shredded Cabbage
}

\author{
Kaoru KohyAma $^{1 *}$, Yuko TAKeZAWA ${ }^{1}$ and Atsushi TAKADA ${ }^{2}$ \\ ${ }^{1}$ National Food Research Institute, 2-1-12 Kannondai, Tsukuba, Ibaraki 305-8642, Japan \\ ${ }^{2}$ Miura Peninsula Region Office of Kanagawa Agricultural Technology Center, 3002 Shimomiyata, Hatsuse-cho, Miura, Kanagawa \\ 238-0111, Japan
}

Received July 8, 2008; Accepted September 4, 2008

The mechanical properties of cabbages (var. T-520) of various head sizes were evaluated by carrying out a tensile test and a puncture test on fifth leaves. Fracture properties of a strap-shaped specimen that was pulled perpendicular to a secondary vein well correlated to the puncture properties of the mesophyll part. The fracture load, fracture stress, and elastic modulus obtained in parallel tension to the fiber were significantly greater than those for the perpendicular direction. The mechanical properties of the fifth leaves of cabbages were not influenced by head size, although they varied within a leaf and among individual leaves. This observation suggests that head size of cabbages is not important to determine the quality of shredded cabbage; thus, large size is more suitable for the industrial production of shredded cabbage.

Keywords: mechanical properties, Winter-type cabbage, head size, shredded cabbage

\section{Introduction}

A large quantity of cabbage (Brassica oleracea var. capitata) produced in Japan is used for the food industry (Yamamoto, 2007). Among commercial uses for the food industry, shredded cabbage is one of the most consumed cut vegetables in Japan. Processing yield is high in the Winter type cabbages (Yano et al., 1986; Kobayashi, 2006; Yamamoto, 2007), and cabbages with high head density and large size are preferred from the same point of view. We evaluated the effects of head density on the mechanical properties of cabbages (Kohyama et al., in press). The present study attempted to clarify the effect of head size on the mechanical properties of shredded cabbage; i.e., whether the head size changes mechanical properties of shredded cabbage in the same way as yield.

Our previous study (Kohyama et al., 2008) demonstrated that tensile fracture tests of strap-shaped specimens, which serve as a model of shredded cabbage, taken from the fifth leaves of cabbages could express mechanical properties of shredded cabbages. Significant differences were found in relation to the direction of tension (perpendicular or parallel to fibers). When a strap-shaped specimen is pulled perpen-

*To whom correspondence should be addressed. Email:kaoruk@affrc.go.jp dicular to a secondary vein, the mesophyll part is broken; in contrast, when it is tensioned parallel to a secondary vein, the fracture properties were related to the vein properties. We here discuss the effects of cabbage head size on qualities required for the industrial production of shredded cabbage.

The other methodology in literature to evaluate cabbage leaves is the puncture resistance test (Yano et al., 1986; 1990; Saito et al., 2000), where a mesophyll part of cabbage leaf is pressed with a cylindrical plunger (diameter of 2-5 $\mathrm{mm}$ ) at a constant speed, and the resistant load is measured. Yano et al. (1986) succeeded in breeding suitable varieties for shredded cabbage in terms of puncture resistance. A puncture test and tensile test perpendicular to the veins were conducted on specimens from the same part of a leaf and the results were compared. We discuss the relationship between the mechanical parameters of cabbage mesophyll obtained from the different methods.

\section{Materials and Methods}

Samples Cabbages (T-520, Takii and Co. Ltd., Kyoto, Japan) were used. This cultivar belongs to the Winter type, grows slowly, and is suitable for processing (Yamamoto, 2007). When planted in warm regions in Japan, the cabbage can be harvested in spring. The samples were seeded on 22 August 2007, transplanted on 14 September 2007, and har- 
vested on 21 April or 12 May, 2008, at the Miura Peninsula Region Office of Kanagawa Agricultural Technology Center, Kanagawa, Japan. The samples were placed in cardboard boxes and transported to the National Food Research Institute, Tsukuba, Ibaraki, Japan, and tested within 4 days at $20^{\circ} \mathrm{C}$.

Each head was measured for weight, diameters (long and short directions), and height. Head volume and head density were calculated as reported previously (Kohyama et al., 2008). As the fifth leaf from the outside was used for the tensile and puncture tests, the weight of the fifth leaf was measured and the ratio to the weight of the whole head was calculated (Kohyama et al., 2008). We classified the cabbage samples into three categories by size; i.e., small, medium, and large. As T-520 cabbage grows large, the small size tested was equivalent to $2 \mathrm{~L}$ size in the market.

Methods for the mechanical tests Mechanical properties were measured by use of a Universal Testing Machine (Model 5542, Instron, Canton, MA, USA) with an attached load cell of $50 \mathrm{~N}$ at $20^{\circ} \mathrm{C}$ (Kohyama et al., 2008).

The fifth leaf from the outside was taken and the main vein was removed. Rectangular specimens $(10 \mathrm{~mm}$ in width and $60 \mathrm{~mm}$ in length parallel or perpendicular to a secondary vein) as shown in Fig. 1A were tensioned in the direction of the big arrow in Fig. 1B at a constant speed of $250 \mathrm{~mm} / \mathrm{min}$ until fracture (Kohyama et al., 2008). Both ends ( $25 \mathrm{~mm}$ each) of the specimen were clamped as shown in Fig. 1B; thus, the initial size of the specimen was $10 \times 10 \mathrm{~mm}$. The thickness and width of the each fractured section were measured with calipers (CD-15, Mitsutoyo, Kawasaki, Japan). Mechanical stress values were derived based on the cross-sectional area (thickness $\times$ width) of each fractured specimen. Strain was calculated as the ratio of deformation to initial length (10 $\mathrm{mm})$.

A specimen (approximately $50 \times 50 \mathrm{~mm}$ ) taken from a fifth leaf was placed on a stainless ring (external diameter of $55 \mathrm{~mm}$ and height of $20 \mathrm{~mm}$ ) with a hole of internal diameter of $10 \mathrm{~mm}$ and then the specimen was covered with another stainless ring with a hole of internal diameter $18 \mathrm{~mm}$ and height $10 \mathrm{~mm}$ (RP-01, Yamaden, Tokyo, Japan) as shown in Fig. 1C. The center position between the rings was punched in the direction of the big arrow in Fig. 1C (Yano et al., 1986) with a stainless cylindrical plunger (diameter of 3.2 $\mathrm{mm}$ ) at a constant speed of $60 \mathrm{~mm} / \mathrm{min}$ until fracture. Three mesophyll parts of each specimen were pressed and the thickness of each punched hole was measured with calipers. Each specimen was punched in three parts. Stress values were calculated as the load divided by the bottom area of the plunger. Strain was calculated as the ratio of the deformation to the fractured thickness.
Statistics Statistical analyses were performed with an SPSS (ver. 14.0J for Windows) package (SPSS Inc., Chicago, IL, USA). An analysis of variance (ANOVA) was conducted to test effect of head size (3) for head or leaf properties and parameters. The ANOVA design was head size $\times$ individual leaves or heads (nested into size). When the size effect was significant $(p<0.05)$, Tukey's multiple comparison tests were performed following the ANOVA, for comparison of the mean values of three sizes.

Experiments Experiment 1 was conducted to clarify the head size effects on the tensile characteristics. More than 12 specimens for each direction were prepared from a fifth leaf. Five individual heads belonging to each size group (small, medium, and large), which were harvested on April 21, were tested. As statistical analyses, a two-way ANOVA (direction of vein $(2) \times$ individual leaf (15)) was first conducted (Kohyama et al., 2008). As the direction effect was highly significant, tensile parameters for the respective directions were analyzed separately.

Experiment 2 was performed to compare the simple puncture and tensile tests. Direction for tension was perpendicular to the secondary vein. The specimens for both tests were cut from close parts (Fig. 1A), where the distance between the corresponding specimens was less than $50 \mathrm{~mm}$ of a fifth leaf. We tested five or more different sites of each fifth leaf from five individual medium size cabbages harvested on May 12.

\section{Results and Discussion}

Characteristics of the head and the fifth leaf of each group We classified the cabbages by head size. The small, medium (approximately average size in the field), and large groups were within the ranges of 1407-1942, 2271-2603, and 3305-4473 g, respectively. Mean and standard deviation for each size group are shown in Table 1. The large head group was greater in diameter, height, volume, the weight of the fifth leaf, and the weight of inner part, but the three groups were similar in head density, the weight ratio of the fifth leaf, and number of leaves per head. As weight of the fifth leaf increased, the distance between veins also increased. Although the ratio of height to diameter was slightly greater in smaller heads, the cabbage head and leaf shape seemed to be similar regardless of size.

Effects of head size on tensile properties When consumers eat cut cabbages, the mechanical properties of each leaf of the edible part are more important than those in the whole head, because they determine the texture of cut vegetables. Greater head size may be preferred by farmers who prefer less effort and by processors who want to achieve high yield of shredded cabbages (Kobayashi, 2006; Yama- 
A

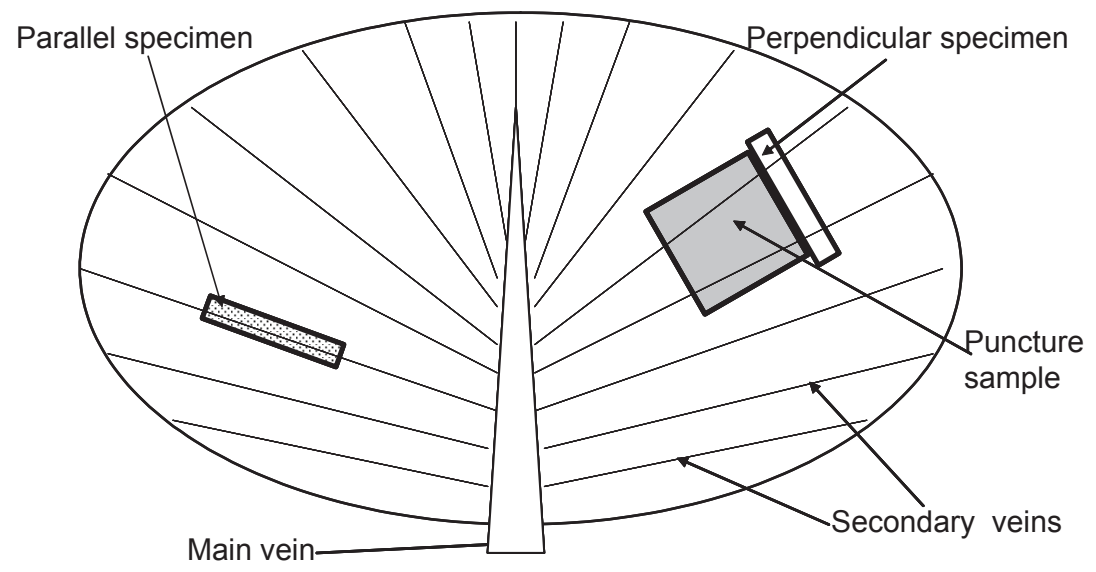

B

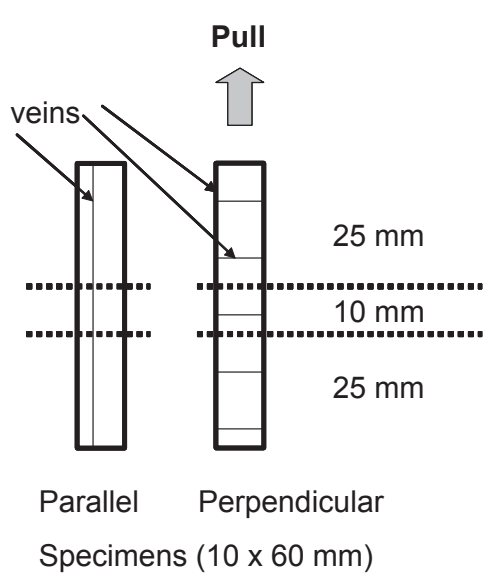

C Upper ring with a $18 \mathrm{~mm}$ hole Punch

Fig. 1. Experimental dimensions for the cabbage tests.

(A) Schematic drawing of specimen preparation from a fifth leaf of cabbage.

(B) Schematic drawing of a parallel and a perpendicular specimens $(10 \times 60 \mathrm{~mm})$ for the tensile test. Middle part $(10 \times 10 \mathrm{~mm})$ was tensioned at a constant speed of $250 \mathrm{~mm} / \mathrm{min}$.

(C) A specimen $(50 \times 50 \mathrm{~mm})$ placed between the support rings for the puncture test. The center position of the ring was pressed with a cylindrical plunger (diameter of $3.2 \mathrm{~mm}$ ) at a constant speed of $60 \mathrm{~mm} / \mathrm{min}$.

moto, 2007). In addition, properties of an individual leaf and shredded cabbage must be acceptable by consumers. As the representative, the fifth leave from the outside was tested by tensile fracture (Kohyama et al., 2008), because the fifth leaf from the outer side of each head of cabbage has the greatest weight among an interval of three leaves (Ninomiya and Sugeno, 1958). The tensile properties of fifth leaves taken from different cultivars differ according to direction of tension and varieties (Kohyama et al., 2008).

Table 2 shows ANOVA results of the fifth leaves of cabbage heads with different sizes for specimens tensioned parallel and perpendicular to the secondary vein. The two-way ANOVA revealed that significant main effects were found for orientation of veins in thickness, fracture load, fracture stress, and elastic modulus $(F(1,498)=11.88,20.97,11.66$, and 48.05, respectively, $p<0.01$ for thickness and $p<0.001$ for the others), whereas the tension directions show similar fracture strains $(F=0.24, p>0.05)$. The former four parameters were greater in the parallel direction than in the perpendicular direction, regardless of head size (Table 2). Thus, we analyzed the tensile properties in the tensile direction separately (Table 2).

Significant main effects of head size were found in fracture load and elastic modulus for both directions, and fracture strain in the perpendicular direction (second column in Table 2). Highly significant effects of individual heads (nested by head size) were observed in fracture strain, fracture load, fracture stress, and elastic modulus $(F(12,262)=9.78,5.55$, 
Table 1. Characteristics of whole head and fifth leaf for three groups classified by head size.

\begin{tabular}{|c|c|c|c|c|c|c|c|}
\hline & $\begin{array}{c}\text { ANOVA } \\
F \text {-ratio }\end{array}$ & $\begin{array}{l}\text { Small } \\
\text { Mean }\end{array}$ & SD & $\begin{array}{l}\text { Medium } \\
\text { Mean }\end{array}$ & SD & $\begin{array}{l}\text { Large } \\
\text { Mean }\end{array}$ & SD \\
\hline Head weight (g) & $51.8^{* * *}$ & 1756 a & $a_{ \pm} 213$ & $2449 b_{ \pm}$ & 126 & $3695{ }^{c} \pm$ & 467 \\
\hline Mean diameter (cm) & $71.9^{* * *}$ & $19.5^{a}$ & $a_{ \pm} 0.7$ & $22.2^{\mathrm{b}} \pm$ & 0.7 & $26.1^{c_{ \pm}}$ & 1.1 \\
\hline Height (cm) & $33.7^{* * *}$ & $13.9^{\mathrm{a}}$ & ${ }^{a} \pm 0.7$ & $15.4^{\mathrm{b}} \pm$ & 0.6 & $17.3^{\mathrm{c}} \pm$ & 0.7 \\
\hline Volume $\left(\mathrm{cm}^{3}\right)$ & $64.5^{\star \star *}$ & $2783^{a}$ & $a_{ \pm} 294$ & $3982 b_{ \pm}^{b}$ & 348 & $6167^{c}{ }^{c}$ & 691 \\
\hline Height/diameter ratio & $4.1^{*}$ & $0.715 b$ & $\mathrm{~b}_{ \pm} 0.030$ & $0.694{ }^{\mathrm{ab}_{ \pm}}$ & \pm 0.020 & $0.665^{a} \pm$ & 0.033 \\
\hline Head density $\left(\mathrm{g} / \mathrm{cm}^{3}\right)$ & 0.6 & 0.631 & \pm 0.035 & $0.619 \pm$ & 0.069 & $0.599 \pm$ & 0.023 \\
\hline Weight of fifth leaf $(\mathrm{g})$ & $11.9^{* *}$ & $46.5^{\mathrm{a}}$ & $a_{ \pm} 10.2$ & $53.1^{\mathrm{ab}_{ \pm}}$ & \pm 8.6 & $87.6^{b} \pm$ & 20.9 \\
\hline Ratio of fifth leaf to head (\%) & 1.5 & 2.63 & \pm 0.39 & $2.17 \pm$ & 0.38 & $2.37 \pm$ & 0.49 \\
\hline Weight of sixth leaf and inner (g) & $43.4^{* * *}$ & $1518{ }^{a}$ & $a_{ \pm} 171$ & $2124^{b}{ }_{ \pm}$ & 98 & $3217^{c} c_{ \pm}$ & 466 \\
\hline Number of leaves & 1.1 & 46.0 & \pm 8.2 & $50.4 \pm$ & 2.3 & $50.0 \pm$ & 2.7 \\
\hline
\end{tabular}

Mean \pm standard deviation of five heads.

$*$, **, and $* * *$ indicate significant differences among sizes with $p<0.05, p<0.01$, and $p<0.001$, respectively.

Mean values followed by different alphabetical letters differed significantly $(p<0.05)$ among size groups by Tukey's test.

Table 2. Results of tensile test of the fifth leaf of cabbages.

\begin{tabular}{|c|c|c|c|c|c|c|c|}
\hline & $\begin{array}{l}\text { ANOVA } \\
F \text {-ratio }\end{array}$ & $\begin{array}{l}\text { Small } \\
\text { Mean }\end{array}$ & SD & $\begin{array}{r}\text { Medium } \\
\text { Mean } \\
\end{array}$ & SD & $\begin{array}{l}\text { Large } \\
\text { Mean }\end{array}$ & SD \\
\hline \multicolumn{8}{|c|}{ Parallel to the second vein (character of vein) } \\
\hline Thickness (mm) & 0.63 & 0.86 & \pm 0.31 & 0.81 & 0.42 & 0.88 & 0.54 \\
\hline Fracture strain $(\mathrm{mm} / \mathrm{mm})$ & 0.28 & 0.231 & \pm 0.087 & $0.233 \pm$ & 0.077 & $0.231 \pm$ & 0.085 \\
\hline Fracture load $(\mathrm{N})$ & $5.92^{* *}$ & 7.85 & ${ }^{b} \pm 2.83$ & $6.53{ }^{\mathrm{a}} \pm$ & 2.91 & $7.399^{\mathrm{ab}} \pm$ & \pm 3.60 \\
\hline Fracture stress (MPa) & 1.13 & 0.940 & \pm 0.280 & $0.897 \pm$ & 0.276 & $0.912 \pm$ & 0.275 \\
\hline Elastic modulus (MPa) & $3.68^{*}$ & 6.35 & ${ }^{b} \pm 1.96$ & $5.81^{\mathrm{b}} \pm$ & 1.68 & $5.76{ }^{a} \pm$ & 1.59 \\
\hline \multicolumn{8}{|c|}{ Perpendicular to the second vein (character of the mesophyll) } \\
\hline Thickness (mm) & 2.46 & 0.76 & \pm 0.34 & $0.68 \pm$ & 0.26 & $0.76 \pm$ & 0.30 \\
\hline Fracture strain $(\mathrm{mm} / \mathrm{mm})$ & $5.50^{* *}$ & 0.221 & $a_{ \pm} 0.056$ & $0.223^{a}{ }_{ \pm}$ & 0.078 & $0.257^{b} b_{ \pm}$ & 0.092 \\
\hline Fracture load $(\mathrm{N})$ & $7.57^{* * *}$ & 6.63 & ${ }^{b} \pm 2.58$ & $5.33^{a}{ }_{ \pm}$ & 2.10 & $6.48^{\mathrm{b}} \pm$ & 2.29 \\
\hline Fracture stress (MPa) & 0.43 & 0.857 & \pm 0.298 & $0.813 \pm$ & 0.276 & $0.834 \pm$ & 0.234 \\
\hline Elastic modulus (MPa) & $3.64^{*}$ & 5.20 & ${ }^{b} \pm 1.78$ & $5.17^{\mathrm{b}} \pm$ & 2.04 & $4.52^{\mathrm{a}} \pm$ & 1.29 \\
\hline
\end{tabular}

Mean \pm standard deviation of five individual head $\times$ more than 12 replicates.

Significant differences among head sizes with *, $p<0.05, * *, p<0.01$, and $* * *, p<0.001$.

Mean values followed by different alphabetical letters differed significantly $(p<0.05)$ among size groups by Tukey's test.

5.07, and 3.59, respectively, $p<10^{-4}$ ) in parallel to the vein. Slight individual effects were observed in fracture strain $(F(12,234)=2.17, p<0.05)$, fracture load $(F=2.38, p<0.01)$ and elastic modulus $(F=1.86, p<0.05)$ but were not found in fracture stress $(F=1.57, p=0.101)$ in the perpendicular direction. As previously observed (Kohyama et al., 2008), the fracture properties in the parallel direction were greater than those in the perpendicular direction except for fracture strain, which was similar for the two directions.

Elastic modulus estimated as maximum slope in the tensile stress-strain curve before fracture decreased with increasing head size for both directions (Table 2). The find- ing may relate to curvature of the specimen, which became greater in the smaller cabbage head. The parallel or perpendicular orientation was determined under the assumption that each specimen was a flat rectangle. When the curvature was significantly large, an error arose in tensile direction. The loss of force due to the tensile direction would be greater in specimens prepared from a smaller size head. The lowest fracture load was observed in the medium size cabbages for both directions (Table 2). This may relate to specimen size; initial size was $10 \times 10 \mathrm{~mm}$ between the chucks. The cabbage leaves had homothetic shape in T-520 cultivar (Table 1); thus, the smaller the leaf or head, the shorter the distance be- 
tween veins. A specimen taken from a small cabbage sometimes contained two veins, but that from a large head seldom had more than one vein. In the former case, the fracture load would become high for both tensile directions. Unlike elastic modulus, no significant correlation was observed between tensile fracture property and head size.

Comparison of puncture test and tensile test Figure 2 shows example tensile and puncture curves. As the thickness was less than $1 \mathrm{~mm}$ for the mesophyll part of the cabbage leaf, the breaking strain became about 5 times leaf thickness in the puncture test. The thickness of the fractured part in the tensile test and that in the puncture test were highly correlated $\left(\mathrm{n}=29, r=0.702, p<10^{-4}\right)$.

Figure 3 shows the relationship between fracture load in the tensile and puncture tests. Significant linear correlation was found ( $\mathrm{n}=29, r=0.541, p<0.01)$. A significant correlation was also found in tensile and puncture fracture energies $(r=0.403, p<0.05)$, because the load and energy were highly correlated in both the tensile and puncture tests. Fracture properties of an individual leaf varied greatly; however if we took the close part of mesophyll, the two test methods gave common mechanical properties of mesophyll.

These observations confirmed that the tensile test perpendicular to the secondary vein expressed the fracture proper-
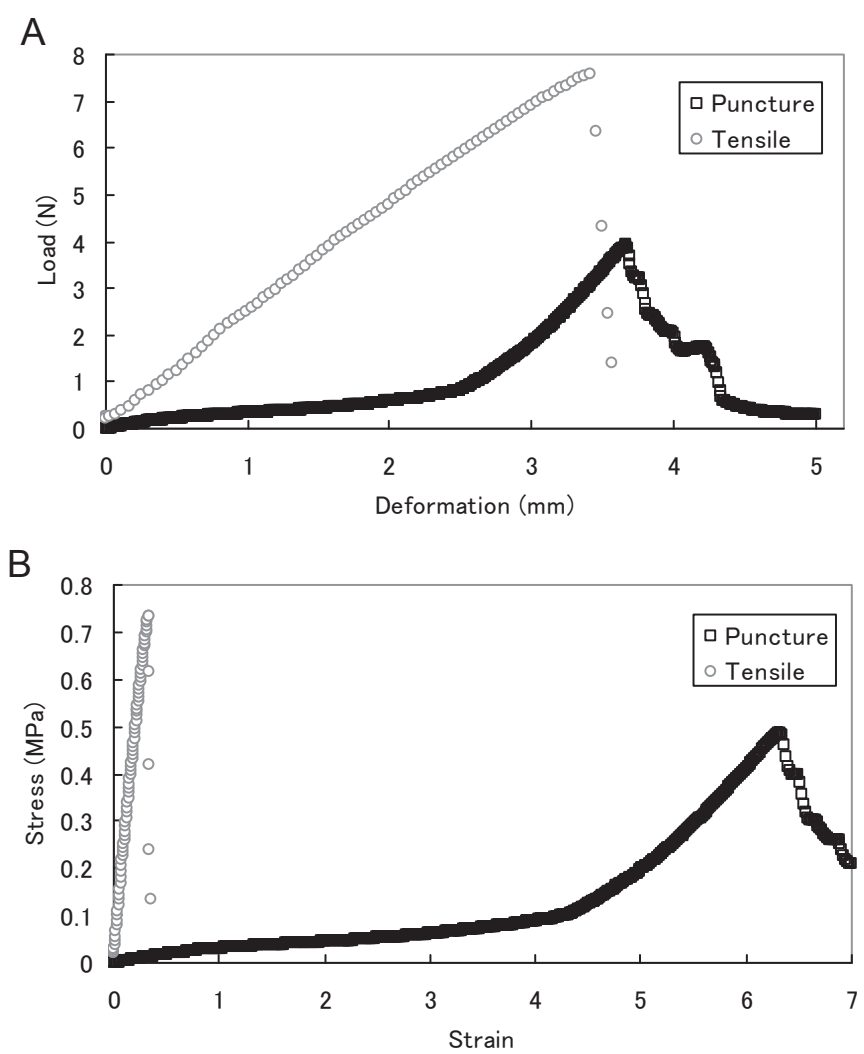

Fig. 2. Example puncture and tensile curves.

(A) Load versus deformation and (B) stress versus strain curves. ties of mesophyll that does not contain veins. The fracture properties significantly differed according to the tensile direction (Table 2); the values were greater in the parallel direction. Therefore, if the stronger part is considered to be important, in the typical case of texture perceived during eating (Kohyama et al., 2008), simple puncture test and tensile test perpendicular to the vein are not appropriate.

As shown in Table 3, the variation among individual cabbage leaves and that within a leaf were significant. The significance values were not high in the tensile test, because of a small number of repetitions. When the tensile test was conducted, the repetition number was more than 12 per each leaf (Table 2). However, the number could not be set sufficiently high in this comparison test to prepare specimens for tensile and puncture testing from a leaf.

Great individual variances shown in Fig. 3 and Table 3 , and highly significant individual differences, especially in parallel tension, may cause the significant differences found in tensile characteristics among any particular head size group. This fact was due to heterogeneity in mechanical properties of cabbage leaves regardless of head size.

Fracture properties generally have greater variances, because fracture itself is a probabilistic phenomenon. The same was also found in the puncture test.

\section{Conclusion}

Mechanical properties of strap-shaped specimens of cabbage fifth leaves could be considered a model of shredded cabbage. The thickness of fractured mesophyll part was correlated between the puncture and tensile tests. None of the fracture properties in a leaf of cabbages correlated with head size. Although larger size head gave specimens with lower elastic modulus, size of cabbage head likely has no effect on fracture properties of the final product after shredding.

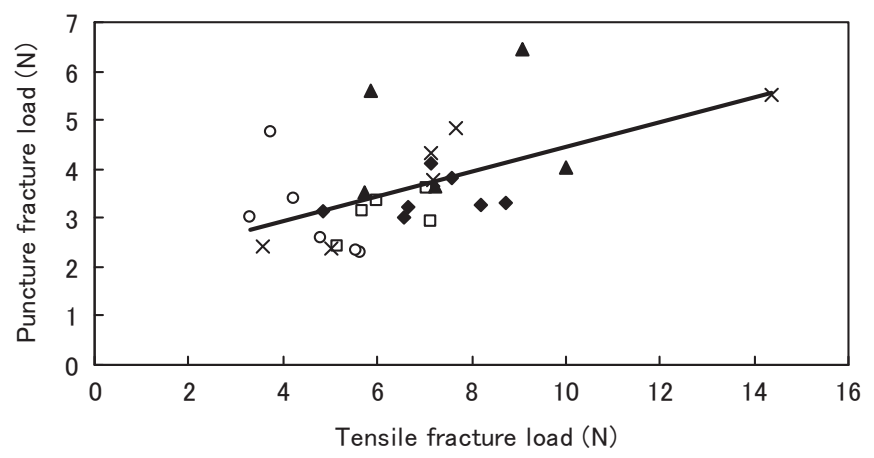

Fig. 3. Relationship between the fracture load values derived from the tensile and puncture tests.

Different symbols represent different individual leaves. Straight line indicates linear regression curve $(r=0.541, p=0.002)$. 
Table 3. Mesophyll properties determined by puncture and perpendicular tensile testing of the same leaves of cabbages.

\begin{tabular}{lcrll}
\hline & ANOVA $F$-ratio & & \\
& Individual Position & Mean & SD \\
\hline \hline Puncture test & & & & \\
Thickness $(\mathrm{mm})$ & $21.9^{* * *}$ & $12.7^{* * *}$ & 0.56 & \pm 0.22 \\
Fracture deformation $(\mathrm{mm})$ & $3.0^{*}$ & $2.2^{* *}$ & 3.127 & \pm 0.453 \\
Fracture strain $(\mathrm{mm} / \mathrm{mm})$ & $8.9^{* * *}$ & $6.6^{* * *}$ & 6.324 & \pm 2.317 \\
Fracture load $(\mathrm{N})$ & $35.3^{* * *}$ & $13.6^{* * *}$ & 3.63 & \pm 1.12 \\
Fracture energy (mJ) & $29.4^{* * *}$ & $13.0^{* * *}$ & 3.03 & \pm 1.41 \\
Tensile test (perpendicular to the second vein) & & & \\
Thickness (mm) & $3.7^{*}$ & & 0.69 & \pm 0.25 \\
Fracture strain (mm/mm) & $2.8^{*}$ & & 0.256 & \pm 0.075 \\
Fracture load (N) & 2.2 & & 6.58 & \pm 2.22 \\
Fracture stress (MPa) & $4.0^{*}$ & & 0.897 & \pm 0.198 \\
Fracture energy (mJ) & 2.3 & 9.48 & \pm 5.56 \\
Elastic modulus (MPa) & $4.5^{* *}$ & & 4.66 & \pm 1.31 \\
\hline
\end{tabular}

Mean \pm standard deviation of 5 heads $\times$ more than 4 parts of a fifth leaf $\times 3$ replicates for puncture test.

Mean \pm standard deviation of 5 heads $\times$ more than 4 replicates for tensile test.

Significant differences among individual leaf or position within a leaf are indicated with $*, p<0.05, * *, p<0.01$, and $* * *, p<0.001$.

Acknowledgment This work was supported by the program for the Breeding and Integrated Research toward Enhancing Consumption of Domestic Farm Products in the Food Service Industry from the Ministry of Agriculture, Forestry and Fisheries of Japan.

\section{References}

Kobayashi, S. (2006). The trend of vegetable demand by intended purposes and the main issues of domestic production. Nourin Suisan Seisaku Kenkyu (in Japanese), 11, 1-27.

Kohyama, K., Saito, T., Takezawa, Y., Matsumoto, I., and Yoshiaki, H. (2009). Effects of head density of cabbages (Brassica oleracea var. Capitata) on the mechanical properties, Food Sci. Technol. Res., 15, in press.

Kohyama, K., Takata, A., Sakurai, N., Hayakawa, F., and Yoshiaki, H. (2008). Tensile test of cabbage leaves for quality evaluation of shredded cabbage, Food Sci. Technol. Res., 14, 337-344.

Ninomiya, K., and Sugeno, M. (1958). On varietal differences of
Chinese cabbage and cabbage as viewed from the constitution of head. Shizuoka-ken Nogyo Shikenjyo Kenkyu Hokoku (in Japanese), 3, 69-84.

Saito, K., Otoguro, C., Odake, S., Kikuchi, S., Sumino, T., and Kaneko, K. (2000). Relationships between pectic substances and texture of cabbage leaf as a result of low temperature steamheating. ITE Letters Batteries New Technol. Med., 1, 786-791.

Yamamoto, M. (2007). Cabbages for processing and industrial uses. Takii Saizensen (in Japanese), 2007 Autumn, 6-8.

Yano, M., Obama, S., Saijo, R. (1986). Varietal differences in the processing suitability of shredded cabbage. Bulletin of the Vegetable and Ornamental Crops Research Station (in Japanese with English abstract), A14, 103-117.

Yano, M., Saijo, R., Sugawara, W., Ohta, H. (1990). Influence of physical and chemical properties on consumer preference of shredded cabbage. Nippon Shokuhin Kogyo Gakkaishi, 37, 478-483. 